\title{
Estabilidad de cepas nativas de Trichoderma conservadas mediante liofilización
}

\section{Stability of native Trichoderma strains preserved by lyophilization}

\author{
Santos T. Leiva-Espinoza ${ }^{1,2^{*}} \mathbb{D}$, Elgar Hernández Diaz ${ }^{2} \mathbb{D}$, Luz Leonor Mattos Calderon ${ }^{3}$
}

\section{RESUMEN}

En este estudio se evaluó la capacidad de especies del género Trichoderma para mantener sus capacidades reproductivas luego de un procedimiento de liofilización. Durante 90 días se evaluaron la capacidad de producir conidios y la capacidad de germinación de cuatro cepas conservadas a dos temperaturas diferentes y contrastantes entre sí, además de la adición de malto dextrina o agua como agente protector. Luego de 90 días de almacenamiento todas las cepas mostraron altos porcentajes de viabilidad y de producción de esporas. La mayoría de tratamientos redujo su capacidad de producir esporas, sin embargo, la cepa F20M4 produjo 2,88x10 UFC/g luego de 90 días de almacenamiento, sin variar respecto de la evaluación hecha a los 30 días. Se evidencia que las esporas conservan mejor su viabilidad al ser conservadas a $28^{\circ} \mathrm{C}$, aunque en todos los casos se observaron disminuciones en la viabilidad de las esporas. Solo siete tratamientos obtuvieron más del 80\% de viabilidad de esporas luego de 90 días de almacenamiento.

Palabras clave: control biológico, conservación, Trichoderma, liofilización, viabilidad.

\begin{abstract}
In this study, the ability of Trichoderma to maintain its reproductive capacities after a lyophilization procedure was studied. During 90 days, the capacity to produce conidia and the germination capacity of 4 strains conserved at two different and contrasting temperatures were evaluated, in addition to the addition of malt dextrin or water as a protective agent. After 90 days of storage, all the strains showed high percentages of viability and spore production. Most of the treatments reduced their capacity to produce spores, however, the F20M4 strain produced 2.88x107 $\mathrm{CFU} / \mathrm{g}$ after 90 days of storage, without varying with respect to the evaluation made at 30 days. It is evidenced that the spores better conserve their viability when stored at $28^{\circ} \mathrm{C}$, although in all cases decreases in the viability of the spores were observed. Only seven treatments obtained more than $80 \%$ spore viability after 90 days of storage.
\end{abstract}

Keywords: biological control, conservation, Trichoderma, lyophilization, viability.

${ }^{3}$ Universidad Nacional Agraria La Molina, Facultad de Agronomía, Departamento Académico de Fitopatología, Lima, Perú.

"Autor de correspondencia: e-mail: santos.leiva@untrm.edu.pe 


\section{INTRODUCCIÓN}

Una alternativa sostenible para disminuir el impacto ambiental causado por el frecuente uso de agroquímicos en el control de plagas y enfermedades de los cultivos se basa en la utilización de agentes de control biológico. Dentro de estos agentes destacan los hongos del género Trichoderma, reconocidos por su actividad antagónica contra algunos hongos fitopatógenos y por su efecto como promotor de crecimiento en plantas (Benítez et al., 2004; Druzhinina et al., 2010).

Los hongos del genero Trichoderma son los antagonistas más utilizados a nivel mundial debido a su ubicuidad, facilidad para ser aislados y cultivados, a su rápido crecimiento en un gran número de sustratos, a que no son patógenas de plantas y mediante aplicaciones foliares puede prevenir enfermedades en un amplio rango de cultivos. Estos hongos se encuentran en todas las zonas climáticas debido a su notable adaptación (Benítez et al.,2004; Bateman et al., 2005).

Para elaborar formulaciones con microorganismos, las células, las esporas o cualquier parte biológica, primeramente, deben someterse a un proceso de conservación para inducir la latencia y proporcionar estabilidad del producto por un tiempo prolongado. Existen métodos de conservación, tales como congelación, secado o liofilización. Eeste último ha resultado ser el más beneficioso para mantener la viabilidad de los microorganismos y su funcionalidad distintiva (Day y Stacey, 2007). Los microorganismos liofilizados están encerrados en una estructura vítrea, que es muy beneficiosa para el almacenamiento a largo plazo de microorganismos deshidratados, y también se pueden almacenar y ser transportados a temperatura ambiente (Day y Stacey 2007). La adición de crioprotectores es considerada benéfica durante el proceso de liofilización como la malto dextrina que protege contra la desnaturalización de proteínas (Tan et al., 2007). Un producto de control biológico, para tener posibilidades de competir comercialmente con productos químicos, debe tener una vida útil mínima de uno a dos años a temperatura ambiente (Abadias et al., 2000). Por lo tanto, formulaciones de hongos deshidratados son atractivas debido a su larga vida útil, fácil manipulación y almacenamiento a temperatura ambiente (Pedreschi y Aguilera, 1997).

En este orden de ideas, en el presente estudio se evalúa la estabilidad de cinco cepas nativas de hongos del genero Trichoderma basados en la conservación de la capacidad de producir conidios y de viabilidad de conidios liofilizados conservados a diferentes temperaturas.

\section{MATERIALES Y METODOS}

Adquisición de Trichoderma spp.

Se utilizaron cinco cepas de Trichoderma spp: IP3M2C4, BIF7-C3, F20M4, BIPF5-C2D2 y AP1M5-C1. Estas cepas fueron aisladas de agroecosistemas de cacao de las provincias de Bagua y Utcubamba como parte de un estudio de diversidad.

Estas cepas se conservaron a $4^{\circ} \mathrm{C}$ en el laboratorio de Entomología y Fitopatología de la Universidad Nacional Toribio Rodríguez de Mendoza de Amazonas.

\section{Diseño experimental}

Para esta investigación se utilizó un DCA (diseño completamente al azar) con un arreglo factorial $5 \mathrm{~A} x$ 3B x 2C, donde A son las cepas de Trichoderma spp. (IP3M2-C4, BIF7-C3, F20M4, BIPF5-C2D2 y AP1M5-C1), B es la temperatura de almacenamiento $\left(4 \mathrm{y} 28^{\circ} \mathrm{C}\right)$ y C los protectores (agua, malta), para un total de 20 tratamientos con tres repeticiones cada uno. El detalle de tratamientos se describe en la Tabla 1. 
Tabla 1. Descripción de tratamientos

\begin{tabular}{|c|c|c|c|}
\hline Cepa & Agente Protector & Temperatura & $\mathrm{N}^{\circ}$ de tratamiento \\
\hline IP3M2-C4 & Malta & $4^{\circ} \mathrm{C}$ & 1 \\
\hline IP3M2-C4 & Malta & $28^{\circ} \mathrm{C}$ & 2 \\
\hline IP3M2-C4 & Agua & $4^{\circ} \mathrm{C}$ & 3 \\
\hline IP3M2-C4 & Agua & $28^{\circ} \mathrm{C}$ & 4 \\
\hline BIPF5-C2D2 & Malta & $4^{\circ} \mathrm{C}$ & 5 \\
\hline BIPF5-C2D2 & Malta & $28^{\circ} \mathrm{C}$ & 6 \\
\hline BIPF5-C2D2 & Agua & $4^{\circ} \mathrm{C}$ & 7 \\
\hline BIPF5-C2D2 & Agua & $28^{\circ} \mathrm{C}$ & 8 \\
\hline BIF7-C3 & Malta & $4^{\circ} \mathrm{C}$ & 9 \\
\hline BIF7-C3 & Malta & $28^{\circ} \mathrm{C}$ & 10 \\
\hline BIF7-C3 & Agua & $4^{\circ} \mathrm{C}$ & 11 \\
\hline BIF7-C3 & Agua & $28^{\circ} \mathrm{C}$ & 12 \\
\hline F20M4 & Malta & $4^{\circ} \mathrm{C}$ & 13 \\
\hline F20M4 & Malta & $28^{\circ} \mathrm{C}$ & 14 \\
\hline F20M4 & Agua & $4^{\circ} \mathrm{C}$ & 15 \\
\hline F20M4 & Agua & $28^{\circ} \mathrm{C}$ & 16 \\
\hline AP1M5-C4 & Malta & $4^{\circ} \mathrm{C}$ & 17 \\
\hline AP1M5-C4 & Malta & $28^{\circ} \mathrm{C}$ & 18 \\
\hline AP1M5-C4 & Agua & $4^{\circ} \mathrm{C}$ & 19 \\
\hline AP1M5-C4 & Agua & $28^{\circ} \mathrm{C}$ & 20 \\
\hline
\end{tabular}

\section{Producción de Trichoderma spp. en sustrato sólido}

La producción de Trichoderma spp. se realizó en sustrato de arroz pre-cocido estéril. Se transfirieron $200 \mathrm{~g}$ de arroz en matraces de $500 \mathrm{ml}$, se añadió la suspensión de Trichoderma previamente estandarizada de conidios $\left(3.68 \times 10^{7} \mathrm{UFC} / \mathrm{ml}\right)$. Luego se incubaron a $28^{\circ} \mathrm{C}$ durante 12 días. Los cultivos se agitaron diariamente a mano para mantener las condiciones aeróbicas de Trichoderma spp. y favorecer la conservación de la biomasa en el proceso de liofilización (Grzegorczyk etal., 2018).

\section{Proceso de liofilización}

Pasados los 12 días de incubación y con el sustrato cubierto de micelio y conidios se procedió a liofilizar cada uno de los tratamientos. Para preparar el sustrato para la liofilización fueron transferidos 200 gramos de arroz de Trichoderma spp. matraz de $500 \mathrm{ml}$ y se añadió $20 \mathrm{ml}$ de una solución estéril de maltodextrina (Malta) al 20\% o agua destilada estéril, según los tratamientos descritos en la tabla 1, como agentes protectores de conidios, se homogenizó adecuadamente la mezcla y se secó en frío con el liofilizador de tríada Labconco, en un colector una presión de 0,2 mbar durante $24 \mathrm{~h}$. Posteriormente, los liofilizados se trans- firieron a tubos plásticos de $50 \mathrm{ml}$ de capacidad y almacenados a una temperatura de $4^{\circ} \mathrm{C}$ y $28^{\circ} \mathrm{C}$ según los tratamientos descritos.

\section{Concentración de conidios}

La concentración de conidios $\cdot \mathrm{mL}-1$ se determinó mediante la fórmula siguiente de Reyes-Figueroa et al. (2016).

$\mathrm{C}=(\mathrm{Cc})\left(4 \times 10^{6}\right)(\mathrm{Fd} / 80)$

Donde:

$\mathrm{C}=$ Concentración (conidios $\cdot \mathrm{mL}-1)$

$\mathrm{Cc}=$ Promedio de conidios contados en la cámara de Neubauer

$\mathrm{Fd}=$ Factor de dilución .

\section{Estabilidad de Trichoderma spp. en almacenamiento}

Para determinar la vida útil de las cepas de Trichoderma spp. se evaluó la viabilidad expresada como la capacidad de germinación de los conidios formulados durante tres meses de almacenamiento. Las evaluaciones se realizaron mensualmente, la dilución que realizó el conteo de esporas se sembró por triplicado en placas Petri con medio agar PDA y se incubaron a 28 
${ }^{\circ} \mathrm{C}$ durante 18 horas. Se cuantificaron 100 conidios entre germinados y no germinados mediante observación al microscopio de cinco campos ópticos por unidad experimental (Ekesi et al., 1999).

\section{Análisis estadístico}

Todos los análisis se realizaron con el software estadístico Infostat V. 2020. Se realizaron análisis de varianza para determinar la existencia de diferencias entre las medias obtenidas para concentración de conidios y porcentaje de germinación. Cuando se identificaron diferencias estadísticas se procedió a realizar una prueba de comparación de medias, específicamente la prueba $\operatorname{Scott} \operatorname{Knott}(\alpha=0,05)$.

\section{RESULTADOS Y DISCUSIÓN}

Las medias de concentración de conidios producidos por cada uno de los tratamientos mostraron diferencias estadísticas significativas ( $p<0,001)$. Diversas tendencias se observan entre los tratamientos según la cepa evaluada y la temperatura de almacenamiento a 30,60 y 90 días de evaluación (Tabla 2).

En la mayoría de tratamientos se aprecia una disminución de la capacidad de producir conidios, tanto para tratamientos con adición de Malta como de agua destilada. A los 90 días de evaluación las tres cepas que obtuvieron la mayor producción de conidios son las que fueron conservadas a $28^{\circ} \mathrm{C}$ y usando extracto de

Tabla 2. Concentración de conidios durante los tres meses de almacenamiento (medias con letras diferentes son significativamente diferentes, Scott Knott, $\alpha=0,05)$

\begin{tabular}{cccclll}
\hline \multirow{2}{*}{ Cepa } & Agente & \multirow{2}{*}{ protector } & Temperatura & \multirow{2}{*}{ Tratamiento } & \multicolumn{4}{c}{ Concentración de esporas (x10 $\mathbf{~ U F C / g ) ~}$} \\
\cline { 6 - 7 } & & $\mathbf{3 0}$ días & $\mathbf{6 0}$ días & $\mathbf{9 0 ~ d i ́ a s ~}$ \\
\hline IP3M2-C4 & Malta & $4^{\circ} \mathrm{C}$ & 1 & $1,23 \mathrm{~d}$ & $1,1 \mathrm{~d}$ & $0,68 \mathrm{~d}$ \\
IP3M2-C4 & Malta & $28^{\circ} \mathrm{C}$ & 2 & $2,7 \mathrm{~b}$ & $2,07 \mathrm{c}$ & $3,32^{\mathrm{a}}$ \\
IP3M2-C4 & Agua & $4^{\circ} \mathrm{C}$ & 3 & $0,87 \mathrm{~d}$ & $0,73 \mathrm{~d}$ & $0,6 \mathrm{~d}$ \\
IP3M2-C4 & Agua & $28^{\circ} \mathrm{C}$ & 4 & $2,02 \mathrm{c}$ & $1,78 \mathrm{c}$ & $2,55 \mathrm{~b}$ \\
BIPF5- & & & & & & \\
C2D2 & Malta & $4^{\circ} \mathrm{C}$ & 5 & $1,28 \mathrm{~d}$ & $1,28 \mathrm{~d}$ & $0,98 \mathrm{~d}$ \\
BIPF5- & & & & & & \\
C2D2 & Malta & $28^{\circ} \mathrm{C}$ & 6 & $1,85 \mathrm{c}$ & $1,92 \mathrm{c}$ & $1,67 \mathrm{c}$ \\
BIPF5- & & & & & & \\
C2D2 & Agua & $4^{\circ} \mathrm{C}$ & 7 & $1,2 \mathrm{~d}$ & $1,2 \mathrm{~d}$ & $0,65 \mathrm{~d}$ \\
BIPF5- & & & & & & \\
C2D2 & Agua & $28^{\circ} \mathrm{C}$ & 8 & $2,07 \mathrm{c}$ & $1,45 \mathrm{~d}$ & $1,55 \mathrm{c}$ \\
BIF7-C3 & Malta & $4^{\circ} \mathrm{C}$ & 9 & $2,63 \mathrm{~b}$ & $1,97 \mathrm{c}$ & $2,03 \mathrm{~b}$ \\
BIF7-C3 & Malta & $28^{\circ} \mathrm{C}$ & 10 & $3,63^{\mathrm{a}}$ & $2,15 \mathrm{~b}$ & $3,42^{\mathrm{a}}$ \\
BIF7-C3 & Agua & $4^{\circ} \mathrm{C}$ & 11 & $1,63 \mathrm{c}$ & $1,8 \mathrm{c}$ & $0,93 \mathrm{~d}$ \\
BIF7-C3 & Agua & $28^{\circ} \mathrm{C}$ & 12 & $2,73 \mathrm{~b}$ & $2,27 \mathrm{~b}$ & $2,45 \mathrm{~b}$ \\
F20M4 & Malta & $4^{\circ} \mathrm{C}$ & 13 & $3,77^{\mathrm{a}}$ & $1,88 \mathrm{c}$ & $1,77 \mathrm{c}$ \\
F20M4 & Malta & $28^{\circ} \mathrm{C}$ & 14 & $3,68^{\mathrm{a}}$ & $2,87^{\mathrm{a}}$ & $3,17^{\mathrm{a}}$ \\
F20M4 & Agua & $4^{\circ} \mathrm{C}$ & 15 & $3,05 \mathrm{~b}$ & $1,72 \mathrm{c}$ & $1,07 \mathrm{~d}$ \\
F20M4 & Agua & $28^{\circ} \mathrm{C}$ & 16 & $2,88 \mathrm{~b}$ & $2,22 \mathrm{~b}$ & $2,88^{\mathrm{a}}$ \\
AP1M5-C4 & Malta & $4^{\circ} \mathrm{C}$ & 17 & $1,87 \mathrm{c}$ & $1,27 \mathrm{~d}$ & $1,03 \mathrm{~d}$ \\
AP1M5-C4 & Malta & $28^{\circ} \mathrm{C}$ & 18 & $2,87 \mathrm{~b}$ & $2,3 \mathrm{~b}$ & $1,52 \mathrm{c}$ \\
AP1M5-C4 & Agua & $4^{\circ} \mathrm{C}$ & 19 & $1,22 \mathrm{~d}$ & $1,67 \mathrm{c}$ & $1,02 \mathrm{~d}$ \\
gAP1M5-C4 & Agua & $28^{\circ} \mathrm{C}$ & 20 & $1,4 \mathrm{~d}$ & $1,63 \mathrm{c}$ & $2,17 \mathrm{~b}$ \\
\hline
\end{tabular}

Malta como agente protector. Estos resultados difieren a los obtenidos por Grzegorczyk et al. (2018), quienes registran cepas con concentraciones superiores a $7 \times 10^{8} \mathrm{UFC} / \mathrm{g}$ sobre paja de arroz como sustrato de fermentación después del período de incubación de tres meses utilizando malto dextrina como agente protector. Tewari y Bhanu (2004), alcanzaron producciones de conidios de $2,8 \times 10^{8} \mathrm{UFC} / \mathrm{g}$ utilizando paja 
Tabla 3. Porcentajes de germinación de conidios durante los tres meses de almacenamiento (medias con letras diferentes son significativamente diferentes, Scott Knott, $\alpha=0,05)$.

\begin{tabular}{|c|c|c|c|c|c|c|}
\hline \multirow{2}{*}{ Сера } & \multirow{2}{*}{$\begin{array}{c}\text { Agente } \\
\text { protector }\end{array}$} & \multirow{2}{*}{ Temperatura } & \multirow{2}{*}{ Tratamiento } & \multicolumn{3}{|c|}{ Germinación de esporas (\%) } \\
\hline & & & & 30 días & 60 días & 90 días \\
\hline IP3M2-C4 & Malta & $4^{\circ} \mathrm{C}$ & 1 & $60,73 b$ & $60,6 c$ & $58 \mathrm{c}$ \\
\hline IP3M2-C4 & Malta & $28^{\circ} \mathrm{C}$ & 2 & $90,87^{\mathrm{a}}$ & $87,27 \mathrm{a}$ & $84,13^{\mathrm{a}}$ \\
\hline IP3M2-C4 & Agua & $4^{\circ} \mathrm{C}$ & 3 & $58,27 \mathrm{c}$ & $60,4 c$ & $55,8 \mathrm{c}$ \\
\hline IP3M2-C4 & Agua & $28^{\circ} \mathrm{C}$ & 4 & $89,6^{\mathrm{a}}$ & $87,2 \mathrm{a}$ & $85^{\mathrm{a}}$ \\
\hline BIPF5-C2D2 & Malta & $4^{\circ} \mathrm{C}$ & 5 & $58,47 \mathrm{c}$ & $61,07 \mathrm{c}$ & $60 \mathrm{c}$ \\
\hline BIPF5-C2D2 & Malta & $28^{\circ} \mathrm{C}$ & 6 & $89,6^{\mathrm{a}}$ & $84,73 b$ & $79,53 b$ \\
\hline BIPF5-C2D2 & Agua & $4^{\circ} \mathrm{C}$ & 7 & $61,8 b$ & $57,6 \mathrm{c}$ & $59 \mathrm{c}$ \\
\hline BIPF5-C2D2 & Agua & $28^{\circ} \mathrm{C}$ & 8 & $88,6^{\mathrm{a}}$ & $84 b$ & $78,2 b$ \\
\hline BIF7-C3 & Malta & $4^{\circ} \mathrm{C}$ & 9 & $60,8 b$ & $58,93 \mathrm{c}$ & $58,8 \mathrm{c}$ \\
\hline BIF7-C3 & Malta & $28^{\circ} \mathrm{C}$ & 10 & $93,53^{\mathrm{a}}$ & $91,13 \mathrm{a}$ & $90,53 a$ \\
\hline BIF7-C3 & Agua & $4^{\circ} \mathrm{C}$ & 11 & $57,2 \mathrm{c}$ & $57,47 \mathrm{c}$ & $55,8 \mathrm{c}$ \\
\hline BIF7-C3 & Agua & $28^{\circ} \mathrm{C}$ & 12 & $88,8^{\mathrm{a}}$ & $83,67 b$ & $80,93 b$ \\
\hline F20M4 & Malta & $4^{\circ} \mathrm{C}$ & 13 & $62,33 b$ & $62,67 \mathrm{c}$ & $61 \mathrm{c}$ \\
\hline F20M4 & Malta & $28^{\circ} \mathrm{C}$ & 14 & $91,6^{\mathrm{a}}$ & $90,33 a$ & $91^{\mathrm{a}}$ \\
\hline F20M4 & Agua & $4^{\circ} \mathrm{C}$ & 15 & $56,53 \mathrm{c}$ & $59,33 \mathrm{c}$ & $56,8 \mathrm{c}$ \\
\hline F20M4 & Agua & $28^{\circ} \mathrm{C}$ & 16 & $87,4^{\mathrm{a}}$ & $82,8 b$ & $79,2 b$ \\
\hline AP1M5-C4 & Malta & $4^{\circ} \mathrm{C}$ & 17 & $60,73 b$ & $61,2 \mathrm{c}$ & $59,93 \mathrm{c}$ \\
\hline AP1M5-C4 & Malta & $28^{\circ} \mathrm{C}$ & 18 & $89,4^{\mathrm{a}}$ & $89,27 \mathrm{a}$ & $88,4^{\mathrm{a}}$ \\
\hline AP1M5-C4 & Agua & $4^{\circ} \mathrm{C}$ & 19 & $56,67 \mathrm{c}$ & $58,93 \mathrm{c}$ & $60,6 \mathrm{c}$ \\
\hline AP1M5-C4 & Agua & $28^{\circ} \mathrm{C}$ & 20 & $89,93^{\mathrm{a}}$ & $88,27 \mathrm{a}$ & $87,67 \mathrm{a}$ \\
\hline
\end{tabular}

de trigo como sustrato de fermentación. Por su parte, Witkowska et al. (2016) alcanzaron $1,7 \times 10^{8}$ y $5,3 \times 10^{8}$ UFC/g al evaluar el efecto de la liofilización sobre la supervivencia y la cinética del crecimiento de cepas de Trichoderma. En ambos estudios se alcanzaron concentraciones superiores a las más altas registradas en nuestro estudio. Eesto podría deberse a diversas causas, entre las más probables podrían destacarse el diferente sustrato de concentración y la diferente capacidad de producción de conidios que tienen las cepas diferentes en hongos del género Trichoderma.

Las medias de viabilidad de conidios mostraron diferencias altamente significativas entre los tratamientos evaluados $(\mathrm{p}<0,0001)$.

Todos los tratamientos almacenados a $4{ }^{\circ} \mathrm{C}$ presentaron germinaciones menores al 80\% luego de 90 días de evaluación (Tabla 3). Valores que están por debajo de los márgenes establecidos como estándares nacionales e internacionales de calidad, que sugieren en la viabilidad como límite mínimo de aceptación para productos biológicos (Jenkins y Grzywacz, 2000). Estos resulta- dos contrastan con los obtenidos por Papavizas (1985), que demostró que los conidios de T. viride no lograron mantenerse viables por un tiempo superior a 20 semanas cuando son almacenados a $30^{\circ} \mathrm{C}$.

Para los tratamientos almacenados a $28^{\circ} \mathrm{C}$ se alcanzaron medias por encima del $80 \%$ en la mayoría de los casos (Tabla 3). Esto podría estar relacionado con los excipientes empleados para la liofilización, ya que la Malta se considera una sustancia protectora de secado que tiene un efecto osmorregulador sobre el microorganismo (Teixido et al., 2005; Grzegorczyk et al., 2018).

El retraso en la germinación de los conidios posiblemente se debe a la deshidratación que sufre durante el tiempo de almacenamiento, lo cual aumenta la tensión superficial. Cuando la temperatura es mayor tiene consecuencias negativas en la viabilidad de los microorganismos durante el almacenamiento y es probable que a mayor temperatura exista una mayor reactividad de las moléculas, presentándose reacciones de degradación que pueden ser tóxicas para el microorganismo (Burges, 1998; Chen et al., 2008). En 
nuestro trabajo la incorporación de la Malta aumentó la capacidad de conservar la viabilidad y logró una mejor estabilidad tanto para producción de conidios como para el porcentaje de germinación en ambas temperaturas de conservación.

\section{CONCLUSIONES}

La biomasa de Trichoderma spp. y la viabilidad de los conidios luego del proceso de liofilización está necesariamente relacionada con la temperatura. Basados en la capacidad de producir conidios y la conservación de viabilidad de conidios los tratamientos que mostraron mejores resultados son los que estuvieron almacenados a $28^{\circ} \mathrm{C}$. Por otro lado, los aditivos influyeron en la concentración y viabilidad de conidios luego de la liofilización y almacenamiento. Además, el uso de Malta obtuvo mejores resultados comparados con el agua. Estos resultados confirman el efecto protector que ejercen los excipientes sobre el principio activo, manteniendo su viabilidad durante el tiempo de almacenamiento.

\section{V.AGRADECIMIENTO}

Este estudio fue financiado por el Fondo Nacional de Desarrollo Científico, Tecnológico y de Innovación Tecnológica FONDECYT CONTRATO N 10-2018FONDECYT-BM-ADT-AV

\section{CONFLICTO DE INTERESES}

Los autores declaran no tener conflicto de intereses.

\section{CONTRIBUCIÓN DE LOS AUTORES}

Conceptualización, Santos Leiva y Elgar Hernandez; metodología, Santos Leiva y Leonor Mattos; análisis formal, Elgar Hernandez; Investigación, Elgar Hernandez y Santos Leiva; preparación de manuscrito (borrador), Elgar Hernandez; Revisión y edición de Manuscrito, Santos Leiva y Leonor Mattos; adquisición de fondos, Santos Leiva. Todos los autores han leído y están de acuerdo con la publicación de esta versión del manuscrito.

\section{REFERENCIAS BIBLIOGRÁFICAS}

Abadias, M., N. Teixido, J. Usall, I. Vinas, y N. Magan. 2000. "Solute Stresses Affect Growth Patterns, Endogenous Water Potentials and Accumulation of Sugars and Sugar Alcohols in Cells of the Biocontrol Yeast Candida Sake." Journal of Applied Microbiology 89 (6): $1009-17$. DOI: $10.1046 / \mathrm{j} .1365$ 2672.2000.01207.x.

Bateman, R. P., E. Hidalgo, J. García, C. Arroyo, G. M. Ten Hoopen, V. Adonijah, y U. Krauss. 2005. "Application of Chemical and Biological Agents for the Management of Frosty Pod Rot (Moniliophthora Roreri) in Costa Rican Cocoa (Theobroma Cacao)." Annals of Applied Biology 147 (2): 129-38. DOI: 10.1111/j.1744-7348.2005.00012.x.

Benítez, T., A. M. Rincón, M. Carmen Limón, y A. C. Codón. 2004. "Biocontrol Mechanisms of Trichoderma Strains." International Microbiology : The Official Journal of the Spanish Society for Microbiology 7 (4): 249-60.

Burges, H. D. 1998. Formulation of Microbial Pesticides, Beneficial Microorganisms, Nematodes and Seed Treatments. Dordrecht (Paises Bajos): Springer.

Chen, H., X. Xiao, J Wang, L. Wu, Z. Zheng, y Z. Yu. 2008. "Antagonistic effects of volatiles generated by Bacillus subtilis on spore germination and hyphal growth of the plant pathogen, Botrytis cinerea." Biotechnology Letters 30 (5): 919-23. DOI: 10.1007/s10529-0079626-9.

Day, J. G., y G. N. Stacey. 2007. Cryopreservation and Freeze-Drying Protocols. Totowa (EEUU): Humana Press. DOI: 10.1007/978-1-59745362-2.

Druzhinina, I. S., M. Komoń-Zelazowska, L. Atanasova, V. Seidl, y C. P. Kubicek. 2010. "Evolution and ecophysiology of the industrial producer Hypocrea jecorina (Anamorph Trichoderma reesei) and a new sympatric 
Agamospecies related to it.". PLoS ONE 5 (2): e9191. DOI: 10.1371/journal.pone.0009191.

Ekesi, S., N. K. Maniania, y K. Ampong-Nyarko. 1999. "Effect of temperature on germination, radial growth and virulence of Metarhizium Anisopliae and Beauveria Bassiana on Megalurothrips Sjostedti." Biocontrol Science and Technology 9 (2): 177-85. DOI: 10.1080/09583159929767.

Grzegorczyk, M., A. Kancelista, W. Łaba, M. Piegza, y D. Witkowska. 2018. "The effect of lyophilization and storage time on the survival rate and hydrolytic activity of Trichoderma strains." Folia Microbiologica 63 (4): 433-41. DOI: 10.1007/s12223-0170581-0.

Jenkins, N. E., y D. Grzywacz. 2000. “Quality control of fungal and viral biocontrol agents - assurance of product performance." Biocontrol Science and Technology 10 (6): 753-77. DOI: 10.1080/09583150020011717.

Papavizas, G. C. 1985. "Trichoderma and Gliocladium: Biology, Ecology, and Potential for Biocontrol." Annual Review of Phytopathology 23 (1): 23-54. DOI: 10.1146/annurev.py.23.090185.000323.

Pedreschi, F., y J. M. Aguilera. 1997. "Viability of Dry Trichoderma harzianum Spores under Storage.” Bioprocess Engineering 17 (3): 177-83. DOI: $10.1007 /$ PL00008963.

Reyes-Figueroa, O, C. F. Ortiz-García, M. T. De La Cruz, L. C. Lagunes-Espinoza, y G. Valdovinos-Ponce. 2016. "Especies de Trichoderma Del Agroecosistema Cacao Con Potencial de Biocontrol Sobre Moniliophthora roreri." Revista Chapingo, Serie Ciencias Forestales y Del Ambiente 22 (2): 149-63. DOI: 10.5154/r.rchscfa.2015.08.036.

Tan, C. S, C. W. Ingen, y J. A. Stalpers. 2007. "FreezeDrying Fungi Using a Shelf-Freeze Drier." Methods Mol Biol 368: 119-25. DOI: 10.1007/978-1-59745-362-2_8.
Teixido, N., T.P. Canamas, J. Usall, R. Torres, N. Magan, y I. Vinas. 2005. “Accumulation of the Compatible Solutes, Glycine-Betaine and Ectoine, in Osmotic Stress Adaptation and Heat Shock Cross-Protection in the Biocontrol Agent Pantoea agglomerans CPA-2." Letters in Applied Microbiology 41 (3): 248-52. DOI: $10.1111 / \mathrm{j} .1472$ 765X.2005.01757.x.

Tewari, L., y C. Bhanu. 2004. "Evaluation of AgroIndustrial Wastes for Conidia Based Inoculum Production of Bio-Control Agent: Trichoderma harzianum." Journal of Scientific and Industrial Research 63 (10): 807-12.

Witkowska, D., A. Kancelista, A. Wilczak, R. Stempniewicz, M. Pasławska, M. Piegza, W. Łaba, y M. Szczech. 2016. "Survivability and Storage Stability of Trichoderma Atroviride TRS40 Preserved by Fluidised Bed Drying on Various Agriculture By-Products." Biocontrol Science and Technology 26 (12): 1591-1604. DOI: $10.1080 / 09583157.2016 .1201457$. 